\title{
Role of Bone Sialoprotein-1 and Its Receptor CD44 Variant 6 in Childhood Acute Lymphoblastic Leukemia
}

\author{
Kareman Ahmed Eshra ${ }^{1 *}$, Radwa Mahmoud El- Sharaby ${ }^{2}$, Mohamed Hossny Fouda ${ }^{2}$, \\ Dina Adam Elshahat $\mathrm{Ali}^{2}$ and Shaymaa Mohamed Elrifaey ${ }^{3}$ \\ ${ }^{1}$ Department of Microbiology and Immunology, Tanta University, Egypt \\ ${ }^{2}$ Department of Clinical pathology, Tanta University, Egypt \\ ${ }^{3}$ Department of Pediateric and Neonatology, Tanta University, Egypt \\ *Corresponding author
}

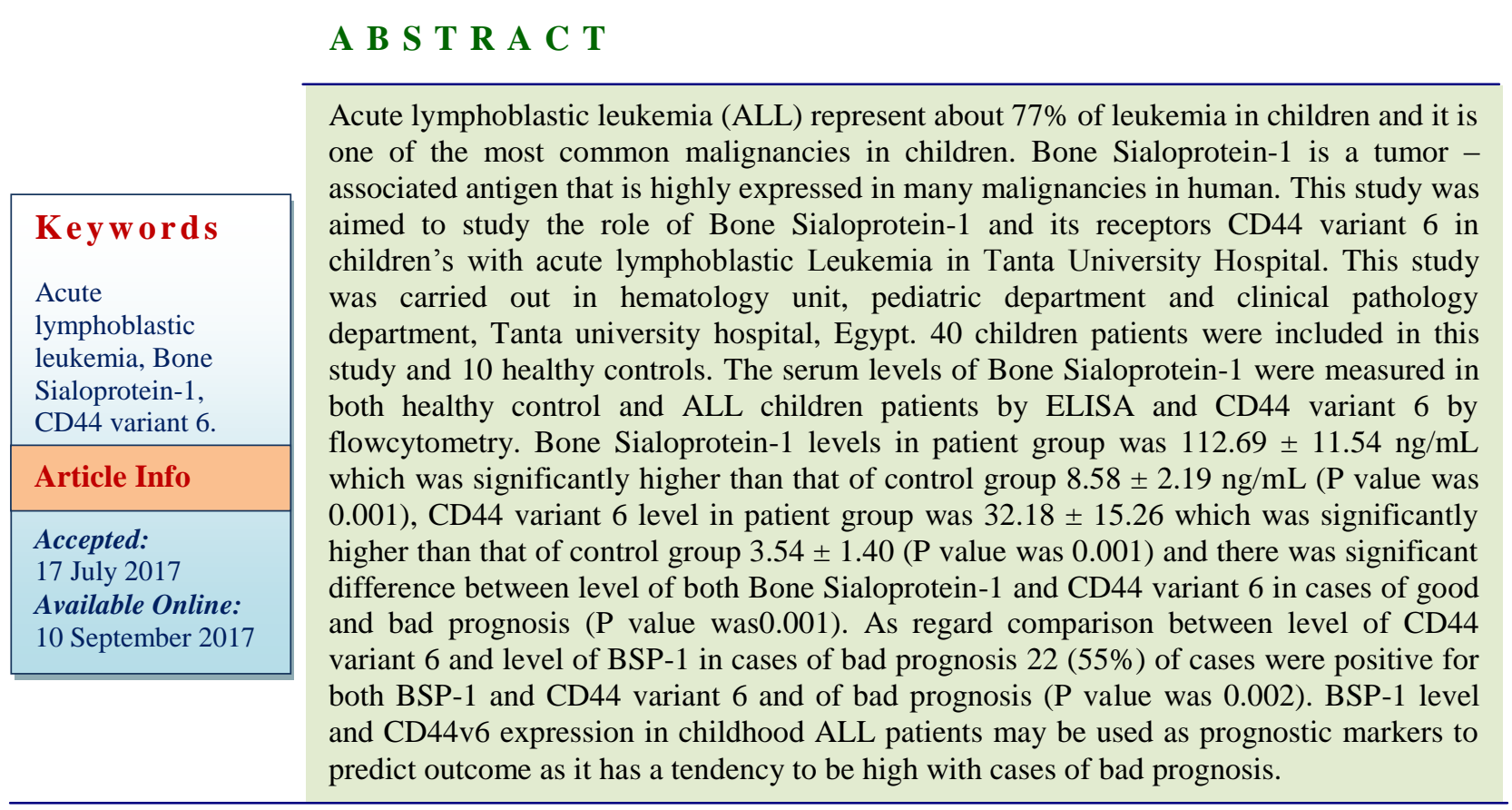

\section{Introduction}

Acute lymphoblastic leukemia (ALL) was defined as amalignancy of lymphoid progenitor cells that proliferate and replace the normal hematopoietic cells of the bone marrow. Then the normal bone marrow elements replaced by These lymphoblasts thus the production of normal blood cells markedly decreased Gowda and Dovat, (2013). The prognosis and the response to treatment of ALL were affected by many prognostic factors Amirghofran et al., (2011). These prognostic factors include age, sex, count of bone marrow blasts, white blood cell (WBC) count and platelet count, cytogenetic abnormalities, extramedullary involvement (EMI), and immune phenotyping Fathi et al., (2012). The different markers expression in childhood ALL were reported and can be used 
as an important source of information for its classification, diagnosis and treatment Kamazani et al., (2013). Immunophenotyping by flowcytometry is considered as one of the most important method used for the diagnosis, classification, staging and monitoring of childhood ALL Craig and Foon, (2008). CD44 is a glycoprotein adhesion molecule; it is expressed by $\mathrm{B}$ and $\mathrm{T}$ lymphocytes, that responsible for cell attachment to extracellular matrix components and adhesion to endothelial cells. Its level of expression change according to type of cell and their activation state Ulrike et al., (2014).CD44 variants $(\mathrm{CD} 44 \mathrm{v})$ are mainly expressed on epithelial cells, There were about 10 types of CD44 variants which detected by PCR (Williams et al., 2013). CD44v6 is reported to play a major role in hematopoietic malignancies pathophysiology and prognosis and to be related to tumor cells metastatic behavior. The increased expression of exons v6 and v9 genes is associated with poor prognosis in non-Hodgkin lymphoma and myeloma (Hao et al., 2012). Bone Sialoprotein-1 (BSP-1) is an adhesive glycoprotein that is secreted and expressed by numerous human malignancies including ALL. It was first detected in bone and later in many other tissues including cartilage, kidney and vascular tissues. BSP-1 plays an important role in different biological processes including inflammation, wound healing and cell survival (Takahashi et al., 2004).

In addition, BSP-1 also might play a protective role in interactions between the epithelial surfaces and the external environment (Rodrigues et al., 2007). It functions in cell adhesion, chemotaxis, macrophage directed interleukin 10 suppression, stress dependent angiogenesis, prevention of apoptosis, anchorage independent growth of tumor cell matrix interactions and cellular signaling through binding with integrin and CD44 receptors (Wai and Kuo, 2004). The various effects produced by BSP-1 is related to its different forms and its many receptors and binding sites, such as multiple integrins (avb3, a9b1, avb7) (Halock and Nilsson, 2006) and CD44 through the v6 and v7 variants (Katagiri et al., 1999). Serum BSP-1 levels were increased in cases with lung, breast, prostate, liver and ovarian cancers, and have been recommended as a prognostic or diagnostic marker (Rittling and Chambers, 2004). In addition, the more increase in the levels of BSP-1 n ALL may give more information on prognosis of ALL in children, Elli et al.,(2012) also, monitoring of these levels along the course of the disease is needed to become sure whether this marker gives more information about bad prognosis of ALL. In our study, we aimed to evaluate the role of Bone Sialoprotein-1and its receptors CD44 variant 6 in children's with acute lymphoblastic Leukemia.

\section{Materials and Methods}

The present study included 40 children's patients admitted to pediatric department (hematology Unit), Tanta University Hospital and 10 healthy control children's, the duration of the study was from June 2016 till February 2017. All the studied patients were subjected to the following: Full history taking, complete clinical examination, Routine laboratory investigations (Complete blood count, Erythrocyte sedimentation rate, Serum Lactate dehydrogenase, Liver and kidney function tests, Bone marrow aspiration, cytochemistry and immunophenotyping for patients only), Detection of BSP-1by ELISA and CD44V6 expression by flowcytometry. Inclusion criteria was newly diagnosed ALL children's patients selected from the pediatric Department (hematology Unit)-Tanta University Hospital and the exclusion criteria were Patients with other types of leukemia or other malignancies. 
Blood Collection and Laboratory Assay: Specimens: Laboratory assays were performed on blood obtained at the time of diagnosis of ALL patients. Whole blood was collected by standard venipuncture in VACUETTE® Blood Collection Tubes (Greiner Bio-One, Austria) containing k2EDTA and containing clot activator/Sep.

Bone marrow aspiration and aspirate examination, cytochemistry and flow cytometry. CBC, liver functions, kidney functions and $\mathrm{LDH}$ were done by routine laboratory methods at hematology unit and clinical chemistry unit, clinical pathology department, Tanta University Hospital, Egypt. Serum samples for determination of BSP-1 were aliquoted and stored frozen at $-20^{\circ} \mathrm{C}$ to avoid loss of human BSP-1 .

\section{Laboratory assay of BSP-1 levels by ELISA}

BSP-1 levels were measured by Human Osteopontin Platinum Enzyme Linked Immunosorbent Assay (ELISA) (BMS2066/ BMS2066TEN, ebioscience). Briefly, BSP-1 present in the sample or standard bound to antibodies adsorbed to the microwells (first incubation). Following incubation unbound biological components were removed by washing and a biotin-conjugated anti-human BSP-1 antibody was added and bound to BSP-1 captured by the first antibody (second incubation). Following this step, unbound biotin conjugated anti-human BSP-1 antibody was removed by washing. Streptavidin-HRP was added and bound to the biotin-conjugated antihuman BSP-1 antibody (third incubation). Following this step, unbound StreptavidinHRP was removed by washing and substrate solution reactive with HRP was added to the wells (fourth incubation). A colored product was formed in proportion to the amount of human BSP-1 present in the sample or standard. The reaction was terminated by addition of acid and absorbance was measured at $450 \mathrm{~nm}$. A standard curve was prepared and human BSP-1 concentrations were determined.

\section{Detection of surface CD44v6 expression by flowcytometry}

For surface staining we first adjust the cell count of each tube to become $1^{*} 10^{\wedge} 6 / \mathrm{ml}$, then the blood were mixed with 10ul of APCconjugated antihuman CD44v6 moAb (R\&D Systems Inc., Clone 2F10, Catalog Number: FAB3660A) and incubated for 25-30 minutes at room temperature in dark place (the same was done for tube of negative isotope control), followed by red cell lysis using B.D lysing solution (diluted 1:9 with D.W) as follow: mix $1 \mathrm{ml}$ of diluted lysing solution to the tested tubes and incubated for 15 minutes at room temperature in dark place, wash twice with PBS buffer, the tubes were centrifuged at $3000 \mathrm{rpm}$ for 5 minutes and the supernatant was discarded, analysis was performed using cell quest software of FACS caliber cytometer.

The percent of cells positive for CD44v6 was determined relative to the negative isotypematched control with cut off value for positivity $5 \%$.

The considered negative (-) If less than $5 \%$ of the cells were labeled, positive (+) if 5 to less than $20 \%$ of cells were labeled, and strong positive $(++)$ when $20 \%$ or more of the cells expressed CD44v6.

\section{Results and Discussion}

Our study was included 40 children patients and 10 healthy controls, their age was ranged from 3 to 12 years old, there were $25(62.5 \%)$ males and $15(37.5 \%)$ females, as regard immunophenotyping $50 \%$ of patients were pre-B-ALL, $2.5 \%$ were mature B-ALL, early 
pre B $2.5 \%$, Common B 35\% (total: $90 \%$ ) and $10 \%$ were T-ALL, as regard results of measuring BSP-1 levels by ELISA in patient group was $112.69 \pm 11.54 \mathrm{ng} / \mathrm{mL}$ which was significantly higher than that of control group $8.58 \pm 2.19 \mathrm{ng} / \mathrm{mL}$ (P value was 0.001 ) as shown in table 1 , as regard CD44 variant 6 level by flowcytometry in patient group was $32.18 \pm 15.26$ which was significantly higher than that of control group $3.54 \pm 1.40$ ( $\mathrm{P}$ value was 0.001 ) as shown in table 2 and out of 40 cases $18(45 \%)$ were high positive results, 16 $(40 \%)$ were positive and $6(15 \%)$ cases were negative as shown in table 3 , and there was significant difference between the level of both Bone Sialoprotein-1 and CD44 variant 6 in cases of good and bad prognosis ( $\mathrm{P}$ value was 0.001) as shown in tables 4 and 5 (cases had good or bad prognosis results according to the response to chemotherapy) (Figs 1-3). As regard comparison between level of CD44 variant 6 and level of BSP-1 in cases of bad prognosis $22(55 \%)$ of cases were positive for both BSP-1 and CD44 variant 6 and of bad prognosis ( $\mathrm{P}$ value was 0.002 ) as shown in table 6 .

Table.1 Measurement of bone sialoprotein-1 by ELISA

\begin{tabular}{|c||c|c|c|c|}
\hline & Cases $(\mathrm{n}=40)$ & Control $(\mathrm{n}=10)$ & T test & P value \\
\hline \hline BSP-1 level by ELISA & $112.69 \pm 11.54 \mathrm{ng} / \mathrm{mL}$ & $8.58 \pm 2.19 \mathrm{ng} / \mathrm{mL}$ & 28.192 & $0.001^{*}$ \\
\hline
\end{tabular}

Table.2 Measurement of CD44 variant 6 by flowcytometry

\begin{tabular}{|l||c|c|c|c|}
\hline & Cases $(n=40)$ & Control $(n=10)$ & T test & P value \\
\hline \hline CD 44 V 6 level by flowcytometry & $32.18 \pm 15.26$ & $3.54 \pm 1.40$ & 8.645 & $0.001^{*}$ \\
\hline
\end{tabular}

Table.3 Numbers and percentage of different levels of CD44 variant 6

\begin{tabular}{|c||c|c|c|c|}
\hline CD 44 V 6 & Cases $(\mathrm{n}=40)$ & Control $(\mathrm{n}=10)$ & $\mathrm{X}^{2}$ & P value \\
\hline \hline High positive (\%) & $18(45 \%)$ & $0(0 \%)$ & & \multirow{2}{*}{26.562} \\
\cline { 1 - 3 } Positive (\%) & $16(40 \%)$ & $0(0 \%)$ & & \\
\cline { 1 - 3 } Negative (\%) & $6(15 \%)$ & $10(100 \%)$ & & \\
\hline
\end{tabular}

Table.4 Comparison between levels of bone sialoprotein-1 by ELISA in cases of good and bad prognosis

\begin{tabular}{|c||c|c|c|c|}
\hline BSP-1 level by ELISA & Cases $(\mathrm{n}=40)$ & Control $(\mathrm{n}=10)$ & $\mathrm{X}^{2}$ & P value \\
\hline \hline Cases of good prognosis $(\%)$ & $16(40 \%)$ & $10(100 \%)$ & \multirow{2}{*}{11.542} & \multirow{2}{*}{$0.001 *$} \\
\hline Cases of bad prognosis $(\%)$ & $24(60 \%)$ & $0(0 \%)$ & & \\
\hline
\end{tabular}

Table.5 comparison between level of CD44 variant 6in cases of good and bad prognosis

\begin{tabular}{|c|c|c|c|c|}
\hline CD 44 V 6 level by flowcytometry & Cases $(n=40)$ & Control $(n=10)$ & $\mathrm{X}^{2}$ & $\mathrm{P}$ value \\
\hline Cases of good prognosis (\%) & $13(32.5 \%)$ & $10(100 \%)$ & \multirow{2}{*}{14.672} & \multirow{2}{*}{$0.001 *$} \\
\hline Cases of bad prognosis (\%) & $27(67.5 \%)$ & $0(0 \%)$ & & \\
\hline
\end{tabular}


Table.6 Comparison between level of CD44 variant 6 and level of bone sialoprotein-1 in cases of bad prognosis

\begin{tabular}{|c||c|c|c|c|}
\hline Cases of bad prognosis & Cases $(\mathrm{n}=40)$ & Control $(\mathrm{n}=10)$ & $\mathrm{X}^{2}$ & $\mathrm{P}$ value \\
\hline \hline Positive cases for CD 44 V 6 & $27(67.5 \%)$ & $0(0 \%)$ & 14.672 & $0.001 *$ \\
\hline Positive cases for BSP-1 & $24(60 \%)$ & $0(0 \%)$ & 11.542 & $0.001 *$ \\
\hline Positive cases for both BSP-1 \& CD 44 V 6 & $22(55 \%)$ & $0(0 \%)$ & 9.821 & $0.002 *$ \\
\hline
\end{tabular}

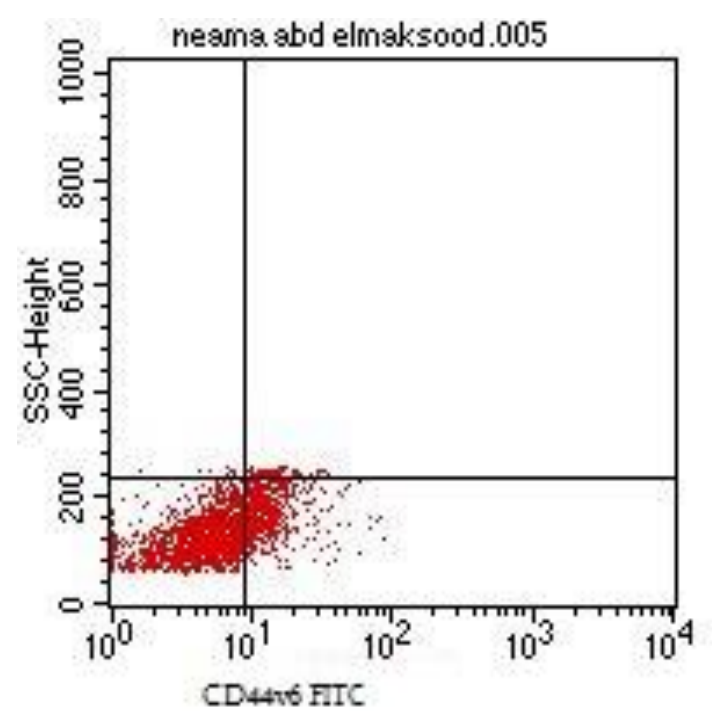

Fig.1 High positive cases for CD44 variant 6

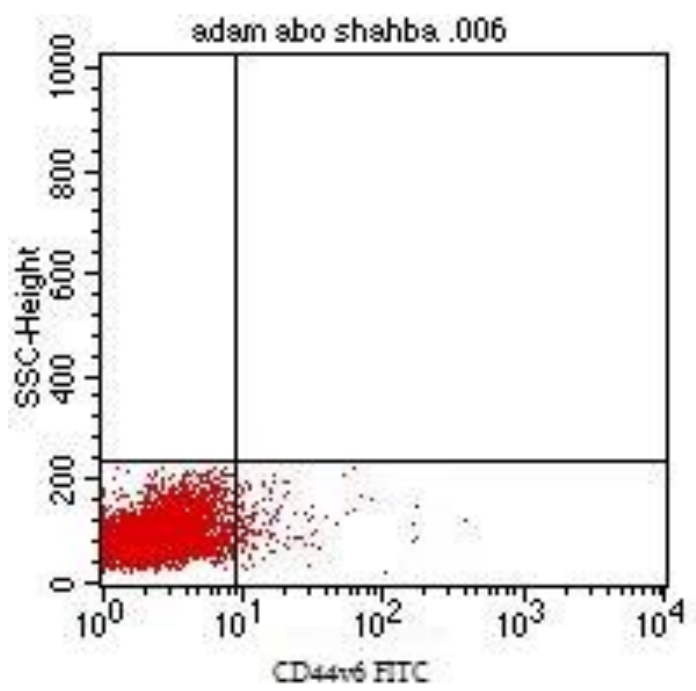

Fig.2 Negative cases for CD44 variant 6

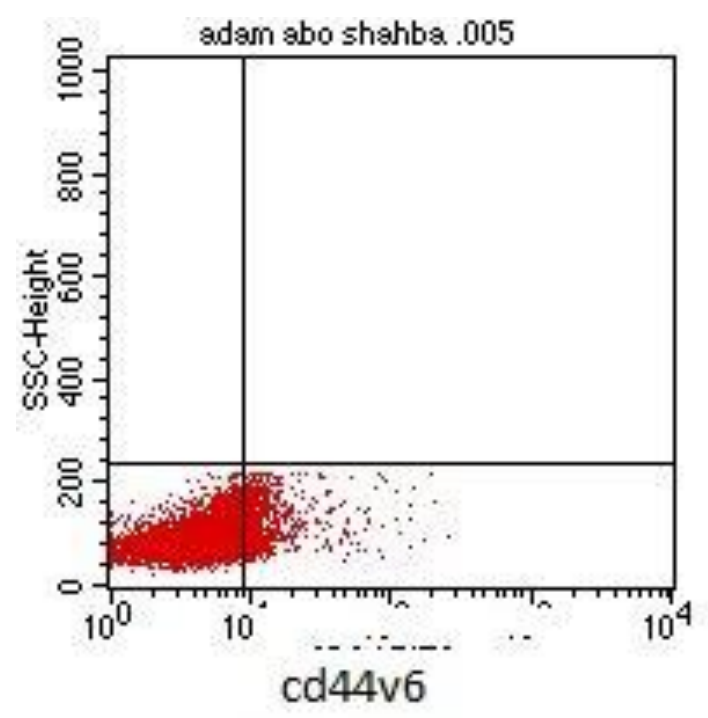

Fig.3 Positive cases for CD44 variant 6 
One of the commonest childhood malignancy is Acute leukemia, with childhood acute lymphoblastic leukemia (ALL) being more common than childhood acute myeloid leukemia (AML). Usually the prognosis of childhood ALL is good, with about $80 \%$ cure rates. Despite this high cure rate relapse still occurs in20-25\% of the cases (Khan et al., 2008). These cases of relapse cannot be sufficiently assessed for their chance of experiencing relapse at diagnosis. For this assessment we need Immunophenotypic and cytogenetic prognostic markers for these patients and these markers also help us to divide cases into high and low risk patients Khan et al., (2008). In our study there were $25(62.5 \%)$ males and $15(37.5 \%)$ females, these results were similar to the result of $\mathrm{Wu}$ et al.,(2010), Reichard et al., (2011) and Stacy and Patrick (2015) who reported increase percentage of male than female in childhood ALL patients. The majority of patients with ALL were of B-lymphoid origin. The B lymphoblastic leukemia is classified as precursor B leukemia (B-ALL) since the blast cells are neoplastic counterparts of normal Bcell precursors Scott (2013). The classification into B- and T-ALL is important for risk stratification and treatment Abdollah et al., (2014).

Immunophenotyping in our study were $50 \%$ of pre-B-ALL, $2.5 \%$ were mature B-ALL, early pre B $2.5 \%$, Common B 35\% (total: $90 \%$ ) and $10 \%$ were T-ALL. This was agreed with the study done by Zhang et al., (2012). In our study, as regard results of measuring BSP-1 levels by ELISA in patient group was $112.69 \pm 11.54 \mathrm{ng} / \mathrm{mL}$ which was significantly higher than that of control group $8.58 \pm 2.19 \mathrm{ng} / \mathrm{mL}$ (P value was 0.001 ) these results agreed with Wang et al.,(2011). Many studies reported that increased BSP-1levels in a variety of malignancies suggested that it might be used as a prognostic marker in these patients as reported by Ang et al., (2005).The increased BSP-1levels in such malignances may be attributed to increased expression and secretion by leukemic blast cells ChaganYasutan et al., (2011). These studies suggested that the expression of BSP-1 was related to the occurrence and development of acute leukemia. Cao et al., (2012) reported that BSP-1 prevent cellular apoptosis and thus play an important role in growth, progression and metastasis of malignancies. In our study there was significant difference between level of both BSP-1 in cases of good and bad prognosis ( $\mathrm{P}$ value was 0.001). This was agreed with Elli et al., (2012) who found that BSP-1 level at the diagnosis may give more information on prognosis in childhood leukemias and lymphoma. As regard CD44 variant 6 level by flowcytometry in patient group was $32.18 \pm 15.26$ which was significantly higher than that of control group $3.54 \pm 1.40$ ( $\mathrm{P}$ value was 0.001 ) and there was significant difference between level CD44 variant 6 in cases of good and bad prognosis ( $\mathrm{P}$ value was 0.001 ). This was in agreement with Bendall et al., (2004), Kyueng et al., (2011) and Todaro et al., (2014) who reported That CD44v6 expression is correlated directly with badprognosis. As regard comparison between level of CD44 variant 6 and level of BSP-1 in cases of bad prognosis $22(55 \%)$ of cases were positive for both BSP-1 and CD44 variant 6 and of bad prognosis ( $P$ value was significant equal to 0.002) and these results were in agreement with Liu et al., (2006) who reported that the level of both of BSP-1 and its receptor CD44 variant 6 were significantly related to cases of bad prognosis, tend to be positive in these cases and thus can predict the bad outcome of cases of childhood ALL.

BSP-1level and CD44v6 expression in childhood ALL patients may be used as prognostic markers to predict outcome as it has a tendency to be high with cases of bad prognosis. 


\section{References}

Abdollah B, Ali G, Lueisa T.2014. Association of cytogenetics and immunophenotype in prognosis of children with acute lymphoblastic leukemia: Literature Review. Reviews in clinical medicine 1: 2-6.

Amirghofran Z, Daneshbod Y, Gholijani N, Esmaeilbeig M.2011. The influence of Bcl-2 and myeloid antigen expression on response to therapy in childhood acute lymphoblastic leukemia. Arch Iran Med 14: 170-174.

Ang C, Chambers AF, Tuck AB, et al., 2005. Plasma osteopontin levels are predictive of disease stage in patients with transitional cell carcinoma of the bladder. BJU Int., 96: 803-805.

Bendall LJ, Nilsson SK, Khan NI, James A, Bonnet C, et al., 2004. Role of CD44 variant exon 6 in acute lymphoblastic leukaemia: association with altered bone marrow localisation and increased tumour burden. Leukemia, 18: 13081311.

Cao DX, Li ZJ, Jiang XO, et al., 2012. Osteopontin as potential biomarker and therapeutic target in gastric and liver cancers. World J Gastroenterol., 18: 3923-3930.

Chagan-Yasutan H, Tsukasaki K, Takahashi

$\mathrm{Y}$, et al., 2011. Involvement of osteopontin and its signaling molecule CD44 in clinic pathological features of adult $\mathrm{T}$ cell leukemia. Leuk. Res., 35: 1484-1490. 11.

Cooper SL, Brown PA2 et al., 2015. Treatment of pediatric acute lymphoblastic leukemia. Pediatr. Clin. North Am., 62: 61-73.

Craig FE and Foon KA. 2008. Flow cytometric immunophenotyping for hematologic neoplasms. Blood, 111(8): 3941-67.

Elli M, Dagdemir A, Bozkurt C, et al., 2012.
Serum osteopontin and CD44 levels in lymphoreticular malignancies in children. Bratisl Lek Listy., 113: 534538.

Fathi M, Amirghofran Z, Shahriari M.2012. Soluble Fas and Fas ligand and prognosis in children with acute lymphoblastic leukemia. Med. Oncol., 29: 20462052.

Gowda C, Dovat S.2013. Genetic targets in pediatric acute lymphoblastic leukemia. Adv Exp Med Biol., 779: 327-340.

Halock DN and Nilsson SK. Osteopontin: a bridge between bone and blood. $\mathrm{Br} J$ Haematol, 134:467-474

Hao Q, Liguan X, Peixue L, Samuel W, Yongkui J. 2012. CD44 ligation with A3D8 antibody induces apoptosis in acute myeloid leukemia cells through binding to $\mathrm{CD} 44 \mathrm{~s}$ and clustering lipid rafts. Cancer Biol. Ther., 13: 12761283.

Kamazani FM, Bahoush GR, Aghaeipour M, Vaeli S, Amirghofran Z.2013. CD44 and CD27 expression pattern in B cell precursor acute lymphoblastic leukemia and its clinical significance. Med Oncol., 30: 359.

Katagiri YU, Sleeman J, Fujii H. CD44 variants but not CD44s cooperate with b-1-containing integrins to permit cells to bind osteopontin independently of arginine-glycine-aspartic acid, thereby stimulating cell motility and chemotaxis. Cancer Res., 1999; 59:219-226.

Khan N, Cisterne A, Devidas M, Shuster J, Hunger SP, et al., 2008. Expression of CD44, but not CD44v6, predicts relapse in children with $\mathrm{B}$ cell progenitor acute lymphoblastic leukemia lacking adverse or favorable genetics. Leukemia \& Lymphoma, 49: 710-718

Kyueng W, Min Y, Ha O, Chan K, Park S, et al., 2011. CD44s and CD44v6 are predominantly expressed in the Non- 
germinal Center B-Cell-like Type of Diffuse Large B-Cell Lymphomas. The Korean Journal of Pathology, 45: 589595.

Liersch R, Gerss J, Schliemann C, et al., 2012. Osteopontin is a prognostic factor for survival of acute myeloid leukemia patients. Blood, 119: 5215-5220.

Liu J, Jiang G, et al., 2006. CD44 and hematologic malignancies. Cell $\mathrm{Mol}$ Immunol., 3: 359-365.

Lymphoblastic Leukemia Allows for the Identification of Impending Relapse: Results of the ALL-BFM-SCT 2003 Trial. JCO 33: 9747v1.

Reichard KK, Kang H, Robinett S.2011. Pediatric B-lymphoblastic leukemia with RUNX amplification: clinicopathologic study of eight cases. Mod Pathol., 24: 1606-1611.

Rittling SR and Chambers AF. 2004. Role of osteopontin in tumour progression. British Journal of Cancer, 90: 1877-1881.

Rodrigues LR, Teixerira JA, Schmitt FL, et al., 2007. The role of osteopontin in tumor progression and metastasis in breast cancer. Cancer Epidemiol Biomarkers Prev., 16: 1087-1097

Scott LM.2013. Lymphoid malignancies: Another face to the Janus kinases. Blood Rev., 27: 63-70.

Takahashi F, Takahashi K, Shimizu K, et al., 2004. Osteopontin is strongly expressed by alveolar macrophages in the lungs of acute respiratory distress syndrome. Lung, 182: 173185.

\section{How to cite this article:}

Kareman Ahmed Eshra, Radwa Mahmoud El- Sharaby, Mohamed Hossny Fouda, Dina Adam Elshahat Ali and Shaymaa Mohamed Elrifaey. 2017. Role of Bone Sialoprotein-1 and Its Receptor CD44 Variant 6 in Childhood Acute Lymphoblastic Leukemia. Int.J.Curr.Microbiol.App.Sci. 6(9): 937-944. doi: https://doi.org/10.20546/ijcmas.2017.609.112 